\title{
Knockdown of Myoferlin Suppresses Migration and Invasion in Clear-Cell Renal-Cell Carcinoma
}

\author{
ALEXANDER COX $^{1 *}$, CHENMING ZHAO $^{1 *}$, YURI TOLKACH ${ }^{2}$, DANIEL NETTERSHEIM ${ }^{3}$, \\ DORIS SCHMIDT ${ }^{1}$, GLEN KRISTIANSEN ${ }^{2}$, STEFAN C. MUELLER ${ }^{1}$, \\ MANUEL RITTER $^{1}$, STEFAN HAUSER ${ }^{1 \#}$ and JOERG ELLINGER ${ }^{1 \#}$ \\ ${ }^{1}$ Department of Urology, University Hospital Bonn, Bonn, Germany; \\ ${ }^{2}$ Institute of Pathology, University Hospital Bonn, Bonn, Germany; \\ ${ }^{3}$ Department of Urology, Urological Research Laboratory, \\ Translational Uro-oncology, University Medical School Düsseldorf, Düsseldorf, Germany
}

\begin{abstract}
Background/Aim: Myoferlin (MYOF) has emerged as an oncogenic protein in various human cancer types. This study was conducted to investigate comprehensively the expression and functional properties of MYOF in clear-cell renal-cell carcinoma (ccRCC) with respect to its value as diagnostic biomarker and therapeutic target. Materials and Methods: $m R N A$ and protein expression of MYOF were assessed by quantitative polymerase chain reaction and immunohistochemistry. siRNA-mediated knockdown of MYOF was performed in the RCC cell line ACHN followed by proliferation, migration and invasion assays. Results: $M Y O F$ $m R N A$ and protein expression were significantly up-regulated in ccRCC. Higher mRNA levels were measured in advanced tumors. MYOF protein expression was increased in tumors with higher histological grades, and those with positive lymph node and surgical margin status. MYOF knockdown led to reduction of migration and invasion in ACHN cells, whereas expression of angiogenesis-associated genes tyrosine-protein kinase receptor-2 (TIE2), angiopoietin 2 (ANG2) and caveolin-1 (CAV1) was up-regulated following knockdown. Conclusion: MYOF may serve as a diagnostic biomarker of tumor progression and a potential therapeutic target in ccRCC.
\end{abstract}

This article is freely accessible online.

\footnotetext{
*These Authors contributed equally to this study.

\#These Authors share senior authorship.
}

Correspondence to: Alexander Cox, MD, Department of Urology, University Hospital Bonn, Venusberg Campus 1, D-53127 Bonn, Germany. Tel: +49 22828714184, Fax: +49 22828714285, e-mail: Alexander.Cox@ukbonn.de, ORCiD ID: 0000-0001-7837-5740

Key Words: Biomarker, immunohistochemistry, knockdown, myoferlin, renal cell carcinoma.
Renal cell carcinoma (RCC) is the most frequent solid renal malignancy and represents $2-3 \%$ of all cancer cases worldwide. Clear cell RCC (ccRCC) is the most common (70-80\%) subtype, followed by papillary $(10 \%)$, chromophobe (5\%) and other subtypes (1). Up to $30 \%$ of all patients will experience tumor progression such as local relapse or distant metastasis after surgery (2). Implementation of tyrosine kinase receptor inhibitors or mechanistic target of rapamycin inhibitors has achieved improvement in therapy of metastatic RCC. In particular, the use of the immune checkpoint inhibitors nivolumab and ipilimumab marked the beginning of a promising era of immunotherapy. However, the response to therapy is still heterogeneous. Thus, it is important to identify novel biomarkers as progression or predictive factors, especially with regard to targeted therapy.

Ferlins are a family of six large ( 200-240 kDa) transmembrane-anchored proteins noted for their role in membrane repair, endocytosis and vesicle trafficking (3). In recent years, ferlins have attracted interest as carriers of disease-causing mutations and the relevance of specific ferlins to carcinogenesis was identified. While mutations of dysferlin (DYSF; formerly FERIL1) cause limb-girdle muscular dystrophy type 2B (4), mutations of otoferlin (OTOF; formerly FERIL2;) were shown to be responsible for an autosomal-recessive form of non-syndromic deafness (5). To date, mutations of the other ferlins such as myoferlin (MYOF; formerly FER1L3), FER1L4, FER1L5 and FER1L6 have not been associated with any distinct disease but their expression seems to play an important role in carcinogenesis. For instance, we revealed that IncRNA FERIL4 is an independent prognostic factor in ccRCC (6). MYOF expression was up-regulated in breast cancer, non-small-cell lung cancer and pancreatic adenocarcinoma (7-10). Recent studies also demonstrated MYOF overexpression in ccRCC $(11,12)$. MYOF has emerging oncogenic impact and 
promotes cancer cell proliferation via different molecular mechanisms. In melanoma cells, down-regulation of $M Y O F$ suppressed vasculogenic mimicry via inhibition of matrix metalloproteinase-2 expression and induction of mesenchymal-to-epithelial transition (13). Furthermore, MYOF sustains the mitochondrial network in colon cancer. $M Y O F$ silencing was shown to lead to reduced oxidative phosphorylation in human colon cancer cells and as a consequence reduced cell growth and increased apoptosis (14). Additionally, MYOF was involved in angiogenesis and has been linked to angiogenesis-associated receptors and proteins such as vascular endothelial growth factor receptor 2 (VEGFR2) and tyrosine-protein kinase receptor-2 (TIE2) (15).

However, the function and molecular mechanisms of MYOF in tumorigenesis have not been fully understood. Little is known about the functional role of MYOF in ccRCC. In this study, we investigated its expression and functional properties in ccRCC cells to identify its value as a diagnostic biomarker and therapeutic target.

\section{Materials and Methods}

The Cancer Genome Atlas (TCGA) analysis. Relative mRNA expression and clinical data from patients with ccRCC were extracted from TCGA by TCGA browser (https://www.cancer.gov/tcga; v0.9.2) to investigate mRNA expression of $M Y O F$. Analysis was carried out using SPSS Statistics v23 (IBM, Ehningen, Germany). The complete cohort comprised 603 samples of patients with available mRNA expression and clinical data (522 ccRCC and 81 benign).

Patients. We examined the expression profile of MYOF mRNA in 82 ccRCC and 44 benign renal tissue samples. Protein expression was assessed in paraffin-embedded RCC tissues on tissue microarrays (TMAs) from patients treated at University Hospital Bonn. The cohort included benign renal tissue and RCC samples (ccRCC $n=142$, benign renal tissue $n=30$ ). Fresh frozen renal tissue samples from patients who underwent radical or partial nephrectomy were collected from the Biobank of the Center for Integrated Oncology Cologne-Bonn. All samples were collected between 1997 and 2014 at the Department of Urology at the University Hospital Bonn. The clinicopathological parameters of the patients are shown in Table I.

Cell culture. The ACHN RCC cell line was obtained from the American Type Culture Collection (ATCC, Manassas, VA, USA). Cells were grown in RPMI 1640 medium supplemented with $10 \%$ inactivated fetal bovine serum, $1 \%$ penicillin-streptomycin antibiotics and $1 \%$ glutamine (all Gibco, Life Technologies, Carlsbad, CA, USA) in an incubator with $5 \% \mathrm{CO}_{2}$ at $37^{\circ} \mathrm{C}$ and $85 \%$ humidity.

SiRNA-mediated knockdown. Transfection of the ACHN cell line was performed with $100 \mathrm{nmol} / \mathrm{l}$ siRNAs (MYOF: HSS120115, Thermo Fisher Scientific, Darmstadt, Germany) using FuGENE ${ }^{\circledR}$ HD Transfection Reagent (Promega, Mannheim, Germany) for 96 hours. The procedures were performed according to the guideline
Table I. Clinicopathological parameters of quantitative polymerase chain reaction ( $q P C R)$ and immunohistochemistry (IHC) cohort.

\begin{tabular}{lcccc}
\hline & \multicolumn{2}{c}{ qPCR } & \multicolumn{2}{c}{ IHC } \\
\cline { 2 - 5 } & $\begin{array}{c}\text { ccRCC } \\
(\mathrm{n}=82)\end{array}$ & $\begin{array}{c}\text { Normal } \\
(\mathrm{n}=44)\end{array}$ & $\begin{array}{c}\text { ccRCC } \\
(\mathrm{n}=142)\end{array}$ & $\begin{array}{c}\text { Normal } \\
(\mathrm{n}=30)\end{array}$ \\
\hline Gender, n (\%) & & & & \\
Male & $59(71.9)$ & $32(72.7)$ & $89(62.7)$ & $21(70.0)$ \\
Female & $23(28.1)$ & $12(27.2)$ & $53(37.3)$ & 9 (30.0) \\
Age, years & & & & \\
Mean & 66.3 & 64.5 & 62.2 & 57.9 \\
Min-max & $38-89$ & $43-86$ & $26-85$ & $28-80$ \\
Pathological stage, $\mathrm{n}$ & & & & \\
pT1 & 41 & n.a. & 59 & n.a. \\
pT2 & 7 & n.a. & 32 & n.a. \\
pT3 & 32 & n.a. & 49 & n.a. \\
pT4 & 2 & n.a. & 2 & n.a. \\
Metastasis, n & & & & \\
Lymph node & 2 & n.a. & 8 & n.a. \\
Distant & 16 & n.a. & 18 & n.a. \\
Grading (WHO 2016), n & & & & \\
G1 & 11 & n.a. & 44 & n.a. \\
G2 & 49 & n.a. & 94 & n.a. \\
G3 & 18 & n.a. & 3 & n.a. \\
G4 & 4 & n.a. & 0 & n.a. \\
\hline
\end{tabular}

n.a.: Not applicable.

of the manufacturer. BLOCK-iT ${ }^{\mathrm{TM}}$ Fluorescent Oligo (Thermo Fisher Scientific) was used as negative control and indicator of transfection efficiency.

RNA isolation. The RNA isolation was performed as described in detail elsewhere (6). In brief, total RNA from fresh frozen tissues was isolated using mirVana ${ }^{\mathrm{TM}}$ miRNA Isolation Kit and treated with DNA-free ${ }^{\mathrm{TM}}$ Kit (both Ambion, Foster City, CA, USA). A NanoDrop 2000 spectrophotometer was used for measurement of RNA quantity (Thermo Fisher Scientific, Wilmington, DE, USA). RNA integrity was proven by agarose gel electrophoresis. cDNA was transcribed using $1 \mu \mathrm{g}$ total RNA (PrimeScript ${ }^{\mathrm{TM}}$ RT reagent Kit with gDNA Eraser; Takara Bio, Saint-Germain-en-Laye, France).

Real-time polymerase chain reaction (PCR). Quantitative real-time PCR (qRT-PCR) was performed to determine the mRNA expression levels of MYOF using SYBR ${ }^{\circledR}$ Premix Ex Taq ${ }^{\text {TM }}$ II with ROX Plus (Takara Bio), $5 \mathrm{ng} / \mu \mathrm{l} \mathrm{cDNA}$ template and $10 \mathrm{pmol} / \mu \mathrm{l}$ of each forward and reverse primer. PCR experiments were performed on an ABIPrism 7900HT Fast Real-Time PCR System (Applied Biosystems, Foster City, CA, USA). The following primer sequences were used: $M Y O F$ (forward AAA-GCT-TGA-GCC-CATTTC-A, reverse TTC-CCA-ATG-CTG-ACT-TCA-AA); caveolin-1 (CAV1; forward ACG-TAG-ACT-CGG-AGG-GAC-A, reverse GCG-TCG-TAC-ACT-TGC-TTC-TC); TIE2; (forward AAG-ACCTAC-GTG-AAT-ACC-AC, reverse GAA-ACA-GAG-GGT-ATACAG-ATG); angiopoietin-2 (ANG2; forward AAG-AGA-AAGATC-AGC-TAC-AGG, reverse CCT-TAG-AGT-TTG-ATG-TGG$\mathrm{AC}$ ). Data were analyzed using Qbase+ (Biogazelle, Ghent, Belgium) with beta-actin $(A C T B)$ and peptidylprolyl Isomerase A 
(PPIA) as reference genes in the $2^{-\triangle \triangle C T}$ algorithm. Both genes were shown to be suitable reference genes for studies in patients with $\operatorname{RCC}(16,17)$.

Western blot. Western blot analyses were made to determine the protein expression of MYOF. Fresh-frozen tumor and normal renal tissues of four patients with ccRCC were investigated. Homogenization of $50 \mathrm{mg}$ tissue was performed in a Precellys 24 (Peqlab, Erlangen, Germany) with $400 \mu$ l Cell Lysis Buffer (Cell Signaling, Cambridge, UK) and protease inhibitor (Complete Mini EDTA-free; Roche, Basel, Switzerland). After determination of protein concentration (BCA Protein Assay Kit; Pierce Biotechnology, Rockford, IL, USA), 35 ng protein per lane was loaded into a NuPAGE 4-12\% denaturating PAA Gel (Life Technologies, Carlsbad, CA, USA) and separated in a Triple-Wide Mini-Electrophoretic Blotting System (XCell4 SureLock Midi-Cell; Life Technologies). The protein extracts were transferred onto 0.2 $\mu \mathrm{m}$ nitrocellulose (iBlot Dry Blotting System; Life Technologies) which was then blocked in 5\% nonfat dry milk in Tris-buffered saline with Tween 20 buffer followed by incubation for $1 \mathrm{~h}$ with primary antibody against MYOF (dilution: 1:300; Sigma-Aldrich, St. Louis, MO, USA). Secondary antibody [anti-rabbit-peroxidase (POD); Biorad, Hercules, CA, USA; anti-biotin-POD; Cell Signaling] conjugated to horseradish peroxidase was used for detection. Chemiluminescent signal was visualized by the LAS 3000 Image Reader (Fujifilm, Tokyo, Japan). ACTB (Sigma-Aldrich) was used as endogenous reference.

Immunohistochemistry. A total of 172 specimens (30 benign renal tissue samples and 142 ccRCC samples) arranged on TMAs were used for this study. In brief, tissues were formalin-fixed and paraffin-embedded, thereupon cut into $4-\mu \mathrm{m}$-thick sections and placed on object plates. After staining with hematoxylin and eosin, representative areas of benign tissues and primary tumor were detected and circled by a pathologist. Three cores of the representative areas each measuring $0.6 \mathrm{~mm}$ in diameter were assembled into TMA blocks using a semiautomatic tissue arrayer (Beecher Instruments, Sun Prairie, WI, USA). TMA sections were assessed again to confirm the histology. The immunohistochemical staining was performed using the Ventana Benchmark automated staining system (Ventana Medical System, Tuscon, AZ, USA). The slides were incubated with primary antibodies against MYOF (dilution 1:50 with Ventana diluent). Signal was detected using an ultraView Universal 3-3'-diaminobenzidine detection kit (Ventana Medical System) conjugated with secondary antibody. Afterwards, the sections were counterstained with hematoxylin and bluing reagent, rehydrated and mounted. Staining was evaluated independently by two readers (AC and YT). Combined membranous and cytoplasmic expression was determined as MYOF is expressed both at the plasma membrane and in the membrane of cytoplasmic endosomes (18). To quantify MYOF protein expression, staining intensities were evaluated according to a score of 0 (no expression) to 3 (strong expression). The cut-off to determine high levels of expression was set at $\geq 2$.

Proliferation assay. EZ4U cell proliferation assay kit (Biomedica Group, Vienna, Austria) was used according to the manufacturer's protocol. The siRNA transfections for proliferation assays were performed in 96-well plates. In each well of a 96-well plate, $3 \times 10^{4}$ ACHN cells (passage 18) were seeded in $200 \mu$ l cell culture medium. siRNA-mediated knockdown of $M Y O F$ was then performed. Afterwards, cells were incubated to adhere and grow for 96 hours. After incubation, $20 \mu \mathrm{l}$ of EZ4U substrate solutions were added. Finally, the resultant solution was incubated for 2 hours until the color of the solution changed from yellow to orange. The absorbance was measured using a microplate reader (Spectra Thermo; Tecan Group, Crailsheim, Germany) at $450 \mathrm{~nm}$ wavelength. Each experiment was repeated at least three times.

Migration and invasion assays. siRNA transfections for migration and invasion assays were performed in 6-well plates. Cells were trypsinized and seeded into migration Boyden chambers 72 hours after transfection. A total of $5 \times 10^{4}$ cells were plated in the upper chamber of migration inserts (VWR, Darmstadt, Germany) containing fetal calf serum-free medium. The lower chamber was filled with medium containing $10 \%$ fetal calf serum for chemotactic attraction. After 24-hour incubation, cells on the upper surface were removed with the help of a cotton swab. Cells invading the lower surface of membrane were fixed with $4 \%$ paraformaldehyde (Merck, Darmstadt, Germany), stained with hematoxylin (Waldeck, Münster, Germany), and washed with water. Membranes were scanned and manually evaluated in four randomly selected fields by counting. Each experiment was repeated at least three times.

The invasion analysis was carried out similarly to the migration experiment except for the use of Matrigel invasion chambers (VWR) and a cell number of $7.5 \times 10^{4} /$ well.

Statistical analyses. Statistical analyses (Mann-Whitney $U$-test and Cox regression analyses) were carried out using SPSS Statistics v23 (IBM, Ehningen, Germany). Optimal cut-off values of mRNA expression data to determine dichotomization for survival analyses (based on consecutive evaluation of all available cut-offs using univariate Cox regression) were calculated using the survMisc package for $\mathrm{R}$ (the $\mathrm{R}$ Foundation for Statistical Computing, Vienna, Austria).

\section{Results}

mRNA expression of MYOF is up-regulated in ccRCC tissue. In the TCGA cohort, significantly higher levels of MYOF mRNA expression were found in ccRCC tumor tissues compared to benign samples $(p<0.001$; data not shown). However, mRNA expression did not correlate with clinicopathological parameters (pT-stage, histological grade, $\mathrm{pN}$-stage, $\mathrm{pM}$-stage) nor survival (all $p>0.05$ ).

In order to validate these findings, we investigated $M Y O F$ mRNA expression in 82 ccRCC and 44 benign renal tissue samples. In our study cohort, MYOF mRNA expression was also significantly up-regulated in tumor tissue (each $p<0.001$, Figure 1A). The receiver operating characteristics analysis identified MYOF as a useful tissue biomarker to discriminate between ccRCC and benign renal tissue: $M Y O F$ mRNA levels discriminated ccRCC from benign tissue with a high specificity of $93.2 \%$ and a mid-range sensitivity of $67.1 \%$ (area under the curve $=0.834$ ). Next, we correlated $M Y O F$ expression levels with clinicopathological parameters (Figure 1). Higher $M Y O F$ mRNA levels were detected in advanced tumors $(p<0.001$; Figure 1B). MYOF mRNA levels did not 
A

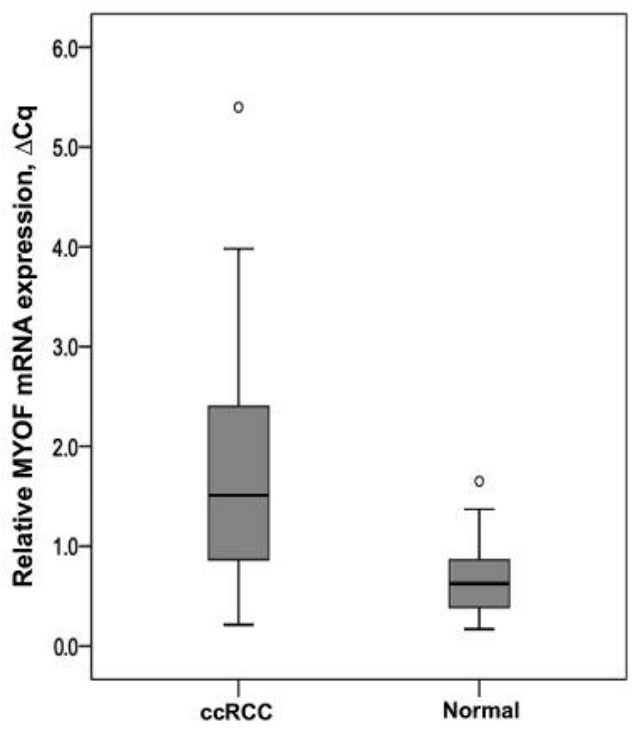

B

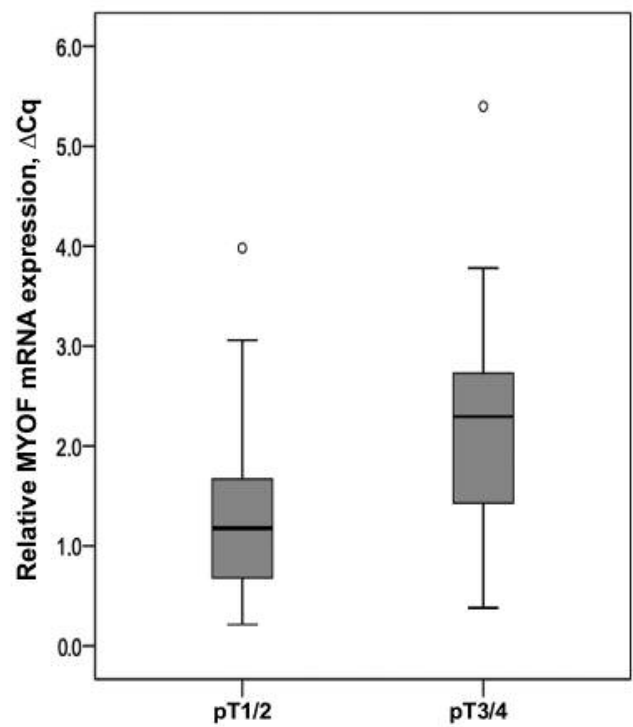

Figure 1. Relative myoferlin (MYOF) mRNA expression. A: MYOF mRNA expression was significantly higher in clear-cell renal-cell carcinoma $(c c R C C)$ compared to control $(p<0.001)$. B: Significantly increased mRNA levels were determined in advanced tumors $(p T 3 / p T 4 ; p<0.001)$. The distribution of relative mRNA expression is shown in box plots. The black bar indicates the median value.

correlate with overall, cancer-specific or progression-free survival (all $p>0.05)$.

Protein expression of MYOF correlates with tumor aggressiveness. We first studied MYOF protein expression by western blot in four ccRCC and corresponding benign renal tissue samples. MYOF protein levels were increased in ccRCC (Figure 2). Interestingly, we detected an additional band with lower molecular weight than the expected. According to Uniprot database there are eight isoforms of MYOF, with molecular weight ranging from 46.69 to $234.70 \mathrm{kDa}$. This likely represents isoform 5 (molecular weight $179 \mathrm{kDa}$ ) (19).

We next investigated the protein expression level using immunohistochemistry in tumor samples from a TMA cohort of 172 patients. Immunohistochemical staining of MYOF in tumor and benign tissues is shown in Figure 3. We confirmed up-regulation of the MYOF protein in ccRCC tissues $(p<0.001$, Figure 4A). Interestingly, increased MYOF expression was positively correlated with tumor grade $p<0.001)$, as well as with the presence of lymph node metastasis $(p=0.004)$ and positive surgical margins ( $p=0.012$; Figures 3 and 4B-D). High levels of MYOF (staining score $\geq 2$ ) showed a trend towards being associated with reduced cancer-specific survival as evaluated by Kaplan-Meier estimates and log-rank test $(\log$-rank $p=0.083$, Figure 4E), however, this was not significant in multivariate analysis. MYOF protein levels were not correlated with overall or progression-free survival.

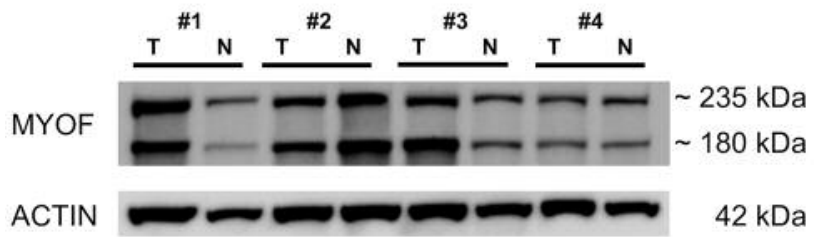

Figure 2. Western blot: myoferlin (MYOF) expression in four clear-cell renal-cell carcinoma $(T)$ and corresponding normal $(N)$ renal tissues. Two isoforms of MYOF were detected. Protein levels were found to be higher in tumor tissues.

siRNA-mediated knockdown suppresses migration and invasion. In order to investigate the functional role of MYOF in tumor progression, we performed siRNAmediated knockdown of $M Y O F$ in the ACHN cell line. $M Y O F$ mRNA and protein expression was significantly down-regulated following siRNA interference $(p \leq 0.001$; Figure $5 \mathrm{~A}$ and $\mathrm{B})$. We observed a significant reduction of migration $(p=0.001)$ and invasion $(p=0.045)$ in ACHN cells compared to the control following knockdown of $M Y O F$ (Figure 5C and D).

As a recent study suggested a decrease in VEGF-mediated cellular proliferation following $M Y O F$ silencing in metastatic ccRCC (20), we additionally investigated the influence of $M Y O F$ knockdown on the angiogenesis-associated genes TIE2, ANG2 and CAV1: Knockdown of MYOF led to an up- 

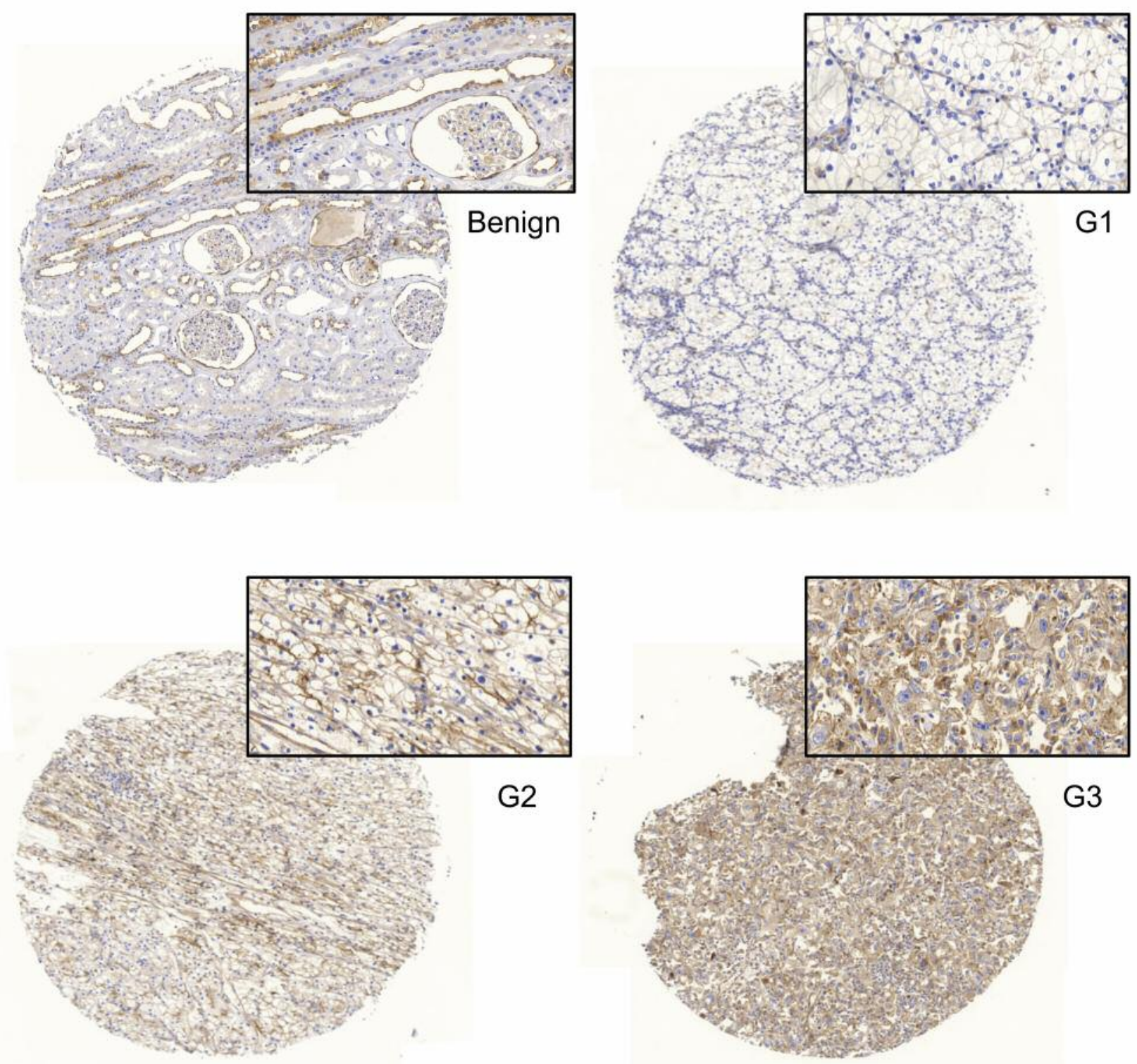

Figure 3. Myoferlin (MYOF) in benign tissue and clear-cell renal-cell carcinoma (ccRCC) of different grades (G) as shown by immunohistochemistry. Representative staining images are shown. MYOF expression significantly increased with increasing grade $(p<0.001)$. Objective magnification: Main image: $\times 10$; inset: $\times 40$.

regulation of these genes (Figure 6) indicating a role of MYOF in angiogenesis.

\section{Discussion}

As transmembrane-anchored vesicle-fusion proteins, ferlins have been described to play an important role in carcinogenesis. In particular, up-regulation of $M Y O F$ was found in various tumor entities as previously stated (7-10). The aim of this study was to investigate MYOF expression and functional properties in ccRCC.
We observed significant up-regulation of $M Y O F$ in ccRCC tissues at the mRNA as well as the protein level. These findings confirm overexpression of MYOF in ccRCC, also recently described by Song et al. (11). Furthermore, increased mRNA and protein expression were correlated to adverse clinicopathological parameters, i.e. mRNA level and advanced stage, protein level and histological grade, lymph node metastasis and positive surgical margins. In summary, the overexpression of MYOF correlates with aspects of tumor aggressiveness. Our findings are therefore concordant with the results obtained for other cancer entities such as lung, breast and pancreatic cancer. 
A

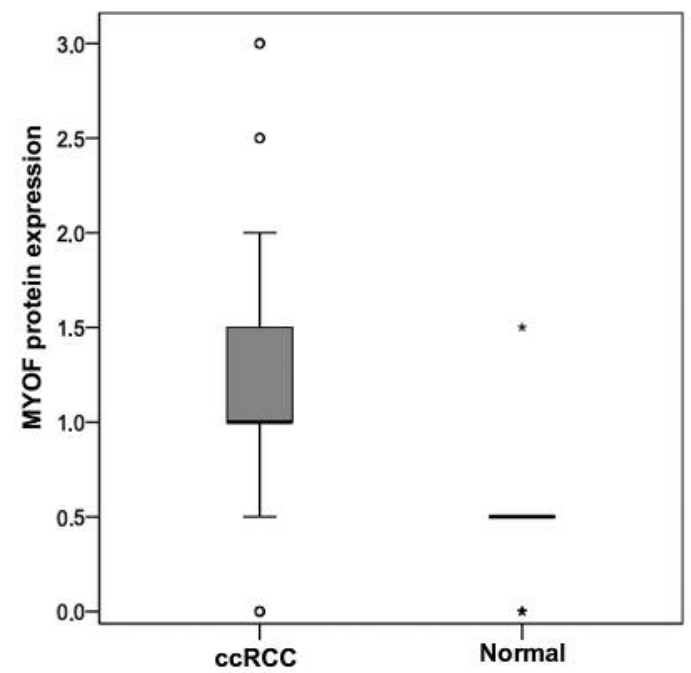

C

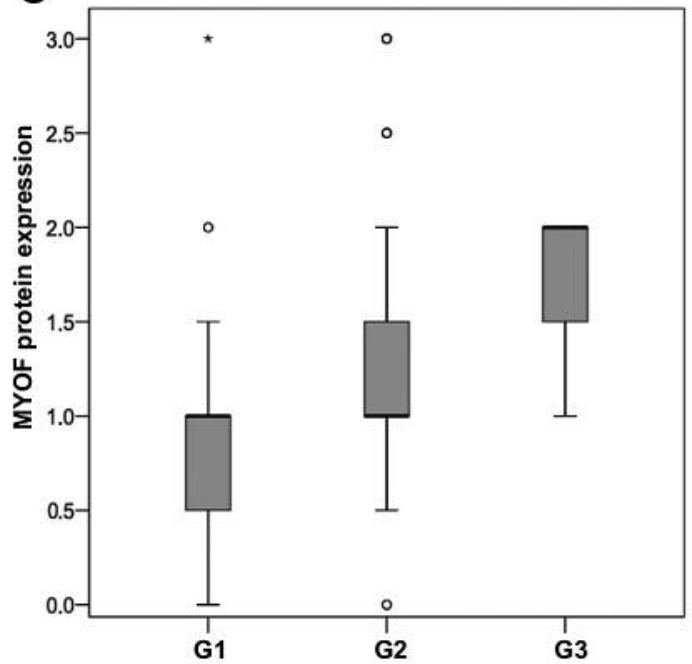

E

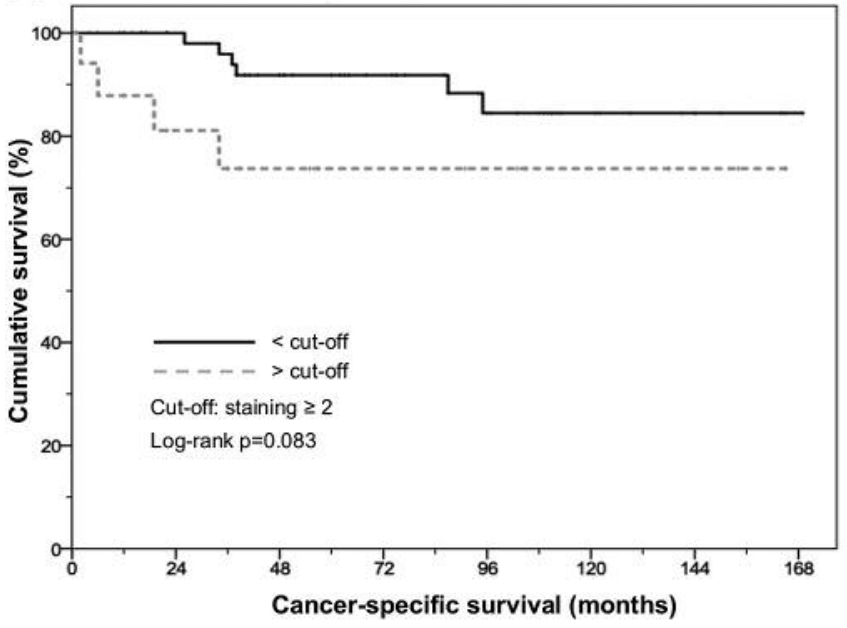

B

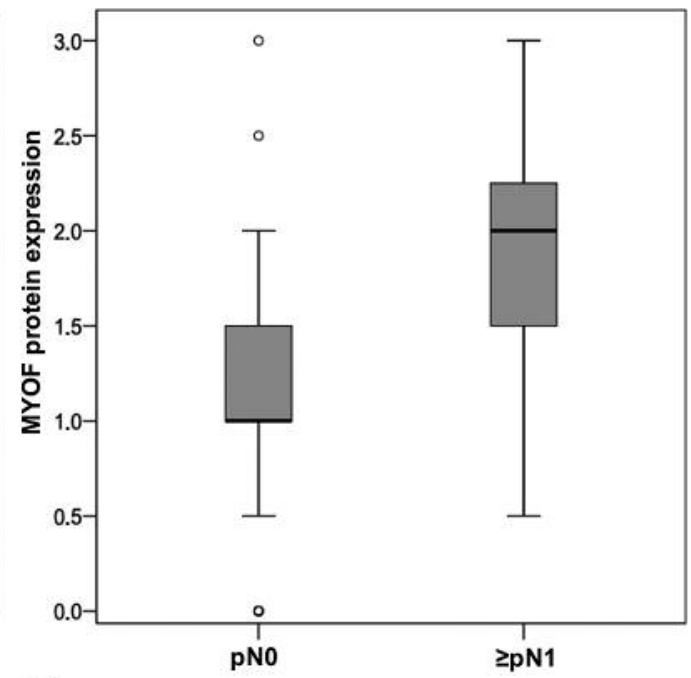

D

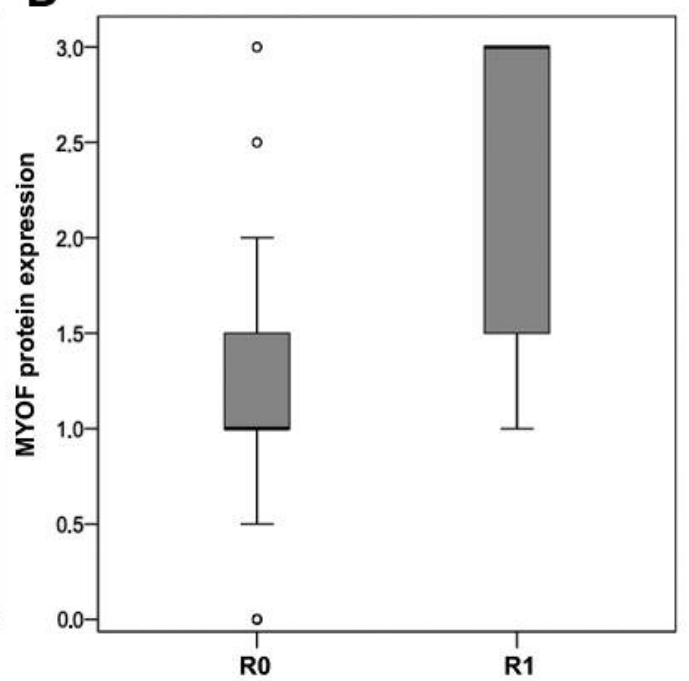

Figure 4. Protein expression of myoferlin (MYOF) in clear-cell renal-cell carcinoma (ccRCC) and corresponding normal tissue (A) and according to nodal status $(B)$, tumor grade $(C)$, and resection margin status $(D)$. Box plots illustrate the median staining score of MYOF protein expression. The black bar indicates the median value. MYOF expression was significantly increased in ccRCC tissue $(p<0.001)$. Increased expression is associated with higher tumor grade $(p<0.001)$, positive lymph node status $(p=0.004)$ as well as with residual tumor $(p=0.012)$. E: Survival analysis using Kaplan-Meier estimates. Using a cut-off of $\geq 2$ (staining intensity of MYOF protein expression), MYOF tended to be predictive of the period of cancer-specific survival in patients with ccRCC (log-rank $p=0.083$ ). 


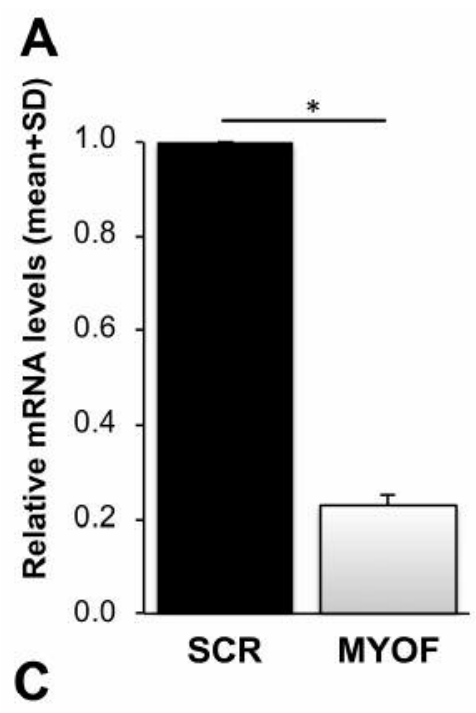

B
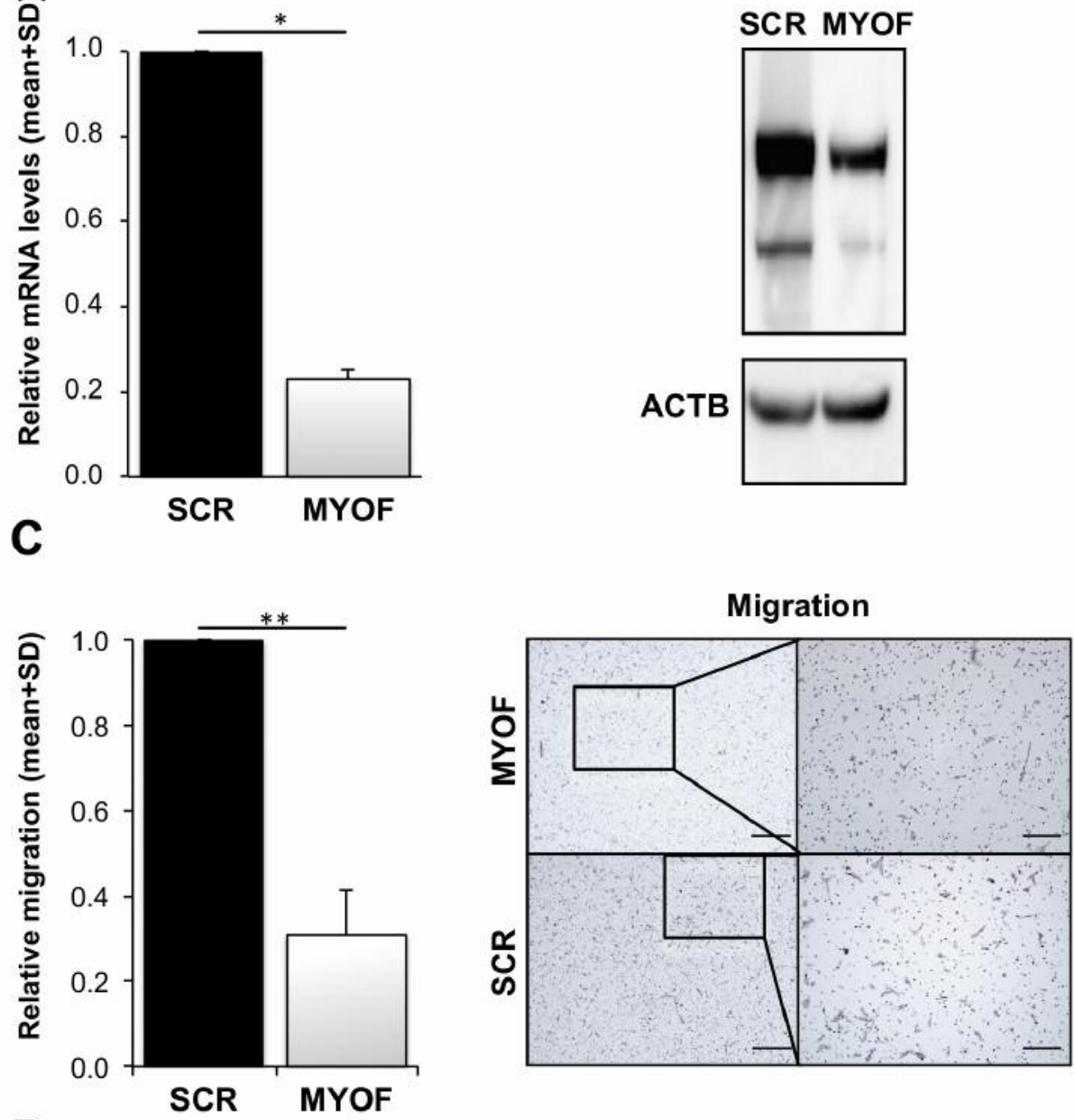

D
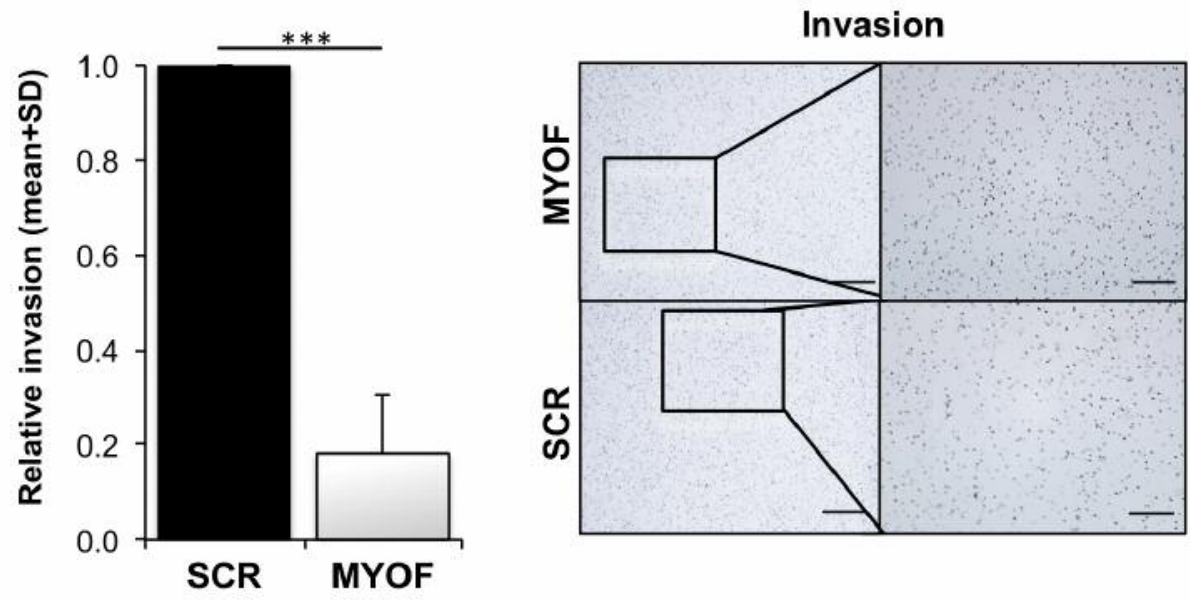

Figure 5. Functional analysis of myoferlin (MYOF) in a clear-cell renal-cell carcinoma cell line, ACHN. In-vitro siRNA-mediated knockdown of $M Y O F$ in the ACHN cell line led to significant reduction of MYOF expression by quantitative polymerase chain reaction (qPCR) (A) as well as in western blot (B). Migration $(C)$ as well as invasion $(D)$ using Boyden chamber assay of the ACHN cell line were also significantly reduced following knockdown of MYOF. Data are the mean \pm standard deviation. Right panel: Objective magnification $\times 4$ and $\times 10$, scale bar 500 and 200 um. Significantly different at $* p<0.001,{ }^{*} p=0.001, *^{* *} p=0.045$. SCR: Scrambled siRNA negative control. 

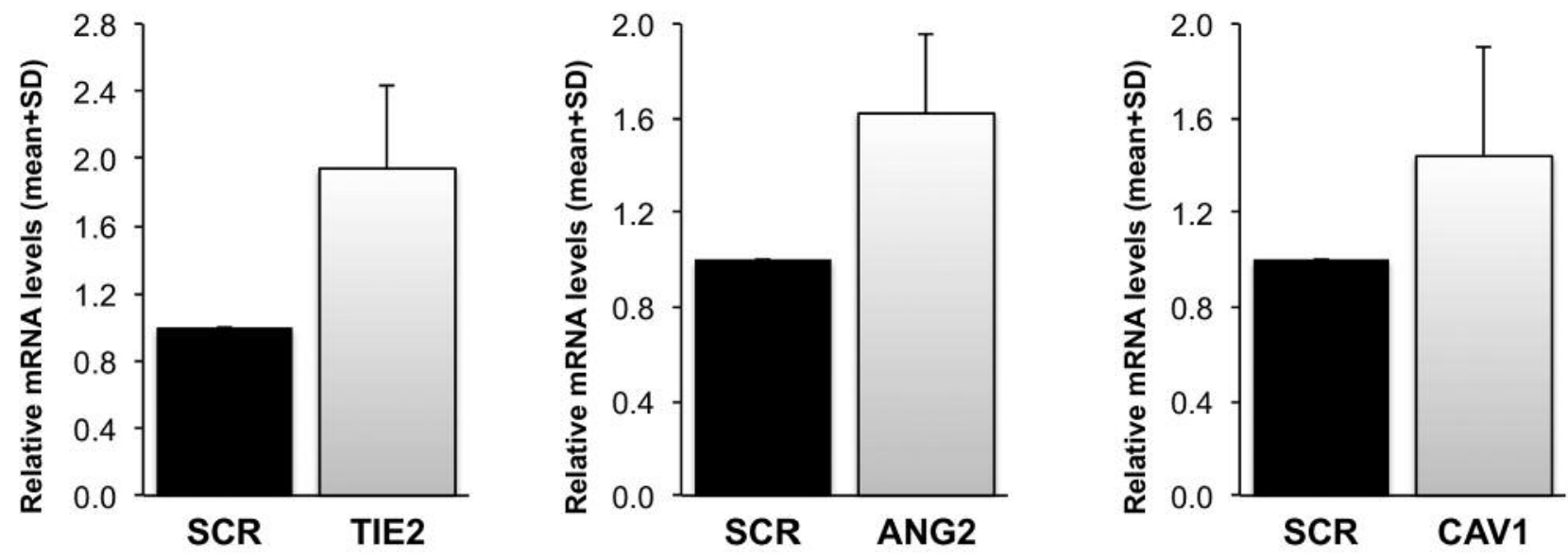

Figure 6. Relative mRNA expression of angiogenesis-associated genes following myoferlin (MYOF) knockdown. siRNA-mediated knockdown of MYOF led to an up-regulation of tyrosine-protein kinase receptor-2 (TIE2), angiopoietin-2 (ANG2) and caveolin-1 (CAV1). Data are the mean \pm standard deviation (differences not significant, $p>0.05$ ).

Involvement of MYOF in cellular signaling pathways has been described, especially an effect on the function of the epidermal growth factor receptor (EGFR) and the VEGFR2. In human breast cancer cells, MYOF regulates EGFR activity as $M Y O F$ silencing blocked EGF-induced migration and epithelial-to-mesenchymal transition (8). A recent study showed a positive relationship between MYOF and EGFR expression in ccRCC, thus indicating that EGFR is involved in the oncogenic properties of MYOF (12). Furthermore, in endothelial cells, MYOF forms a complex with dynamin-2 and VEGFR2 that prevents VEGFR2 polyubiquitination and proteasomal degradation. In $M Y O F$ knock-out mice, loss of protein expression inhibited proliferation and migration (15, 21). Another study examined the expression of VEGFR as well as MYOF in ccRCC; although an inverse correlation of MYOF and VEGFR in ccRCC tissues was observed, knockdown of $M Y O F$ led to down-regulation of VEGFR in metastatic ccRCC cell lines. The loss of MYOF additionally reduced cell confluence, leading the authors to conclude that MYOF affects cell proliferation by regulating VEGFR degradation (20). MYOF also participates in angiogenesis through its interaction with other proteins. In pancreatic ductal adenocarcinoma cells, MYOF knockdown attenuated VEGFA secretion due to an impairment of VEGFA exocytosis, thereby reducing blood vessel density (22). Furthermore, an influence of MYOF on the well-known angiogenic TIE2 receptor was also described. In endothelial cells, MYOF silencing led to reduction of TIE2 receptor expression. (23). Besides these proteins, overexpression of the angiogenesis-associated proteins ANG2 and CAV1 in RCC with influence on tumor progression is well known (2427). Since the influence of MYOF on these proteins in
ccRCC has not yet been investigated, we analyzed the expression of TIE2, ANG2 and CAV1 following knockdown of MYOF. We showed that TIE2, ANG2 and CAV1 expression increased following knockdown of MYOF in ACHN cells. This was accompanied by suppression of migration and invasion indicating that MYOF is able to exert its oncogenic properties in ccRCC by regulating these genes.

\section{Conclusion}

Our study shows that MYOF expression is associated with aggressive ccRCC and affects migration and invasion of tumor cells. Thus, MYOF plays an important role in the carcinogenesis of ccRCC. These findings highlight MYOF both as a biomarker of tumor progression and as a promising target for future treatment of ccRCC.

\section{Conflicts of Interest}

All Authors declare that they have no competing interests. They confirm that the research was conducted in the absence of any commercial or financial relationships.

\section{Authors' Contributions}

JE and SH conceived and designed the study. AC and CZ performed the experiments. YT and GK helped with the evaluation of the immunohistochemistry. DN helped to implement the siRNAmediated knockdown. DS helped with PCR and cell culture. AC, $\mathrm{CZ}$ and JE performed the statistical analyses. All Authors were subsequently involved in data interpretation. AC wrote and drafted the article with the support of all Authors. All Authors read, revised and approved the final version of the article. 


\section{Acknowledgements}

The tissue samples were collected within the framework of the Biobank of the Center for Integrated Oncology Cologne Bonn at the University Hospital Bonn.

\section{Ethical Approval}

All procedures performed in this retrospective study involving human participants were in accordance with the local Ethics Committee (number: 045/17) and with the 1964 Helsinki declaration and its later amendments or comparable ethical standards. This project did not contain any studies with animals.

\section{Informed Consent}

Written informed consent was obtained from all individual patients before enrolment in this study.

\section{References}

1 Ljungberg B, Bensalah K, Canfield S, Dabestani S, Hofmann F, Hora M, Kuczyk MA, Lam T, Marconi L, Merseburger AS, Mulders P, Powles T, Staehler M, Volpe A and Bex A: EAU guidelines on renal cell carcinoma: 2014 update. Eur Urol 67: 913924, 2015. PMID: 25616710. DOI: 10.1016/j.eururo.2015.01.005

2 Stewart GD, O’Mahony FC, Powles T, Riddick ACP, Harrison DJ and Faratian D: What can molecular pathology contribute to the management of renal cell carcinoma? Nat Rev Urol 8: 255265, 2011. PMID: 21487387. DOI: 10.1038/nrurol.2011.43

3 Lek A, Evesson FJ, Sutton RB, North KN and Cooper ST: Ferlins: regulators of vesicle fusion for auditory neurotransmission, receptor trafficking and membrane repair. Traffic 13: 185-194, 2012. PMID: 21838746. DOI: 10.1111/j.1600-0854.2011.01267.x

4 Bansal D, Miyake K, Vogel SS, Groh S, Chen C-C, Williamson R, McNeil PL and Campbell KP: Defective membrane repair in dysferlin-deficient muscular dystrophy. Nature 423: 168-172, 2003. PMID: 12736685 . DOI: $10.1038 /$ nature 01573

5 Yasunaga S, Grati M, Cohen-Salmon M, El-Amraoui A, Mustapha M, Salem N, El-Zir E, Loiselet J and Petit C: A mutation in $O T O F$, encoding otoferlin, a FER-1-like protein, causes DFNB9, a nonsyndromic form of deafness. Nat Genet 21: 363-369, 1999. PMID: 10192385. DOI: $10.1038 / 7693$

6 Cox A, Tolkach Y, Kristiansen G, Ritter M and Ellinger J: The lncRNA Fer1L4 is an adverse prognostic parameter in clear-cell renal-cell carcinoma. Clin Transl Oncol, 2020. PMID: 31965534. DOI: 10.1007/s12094-020-02291-0

7 Amatschek S, Koenig U, Auer H, Steinlein P, Pacher M, Gruenfelder A, Dekan G, Vogl S, Kubista E, Heider K-H, Stratowa C, Schreiber M and Sommergruber W: Tissue-wide expression profiling using cDNA subtraction and microarrays to identify tumor-specific genes. Cancer Res 64: 844-856, 2004. PMID: 14871811. DOI: 10.1158/0008-5472.can-032361

8 Turtoi A, Blomme A, Bellahcene A, Gilles C, Hennequiere V, Peixoto P, Bianchi E, Noel A, De Pauw E, Lifrange E, Delvenne $\mathrm{P}$ and Castronovo V: Myoferlin is a key regulator of EGFR activity in breast cancer. Cancer Res 73: 5438-5448, 2013. PMID: 23864327. DOI: 10.1158/0008-5472.CAN-13-1142
9 Leung C, Yu C, Lin MI, Tognon C and Bernatchez P: Expression of myoferlin in human and murine carcinoma tumors: Role in membrane repair, cell proliferation, and tumorigenesis. Am J Pathol 182: 1900-1909, 2013. PMID: 23499551. DOI: 10.1016/j.ajpath.2013.01.041

10 Wang W-S, Liu X-H, Liu L-X, Lou W-H, Jin D-Y, Yang P-Y and Wang X-L: iTRAQ-based quantitative proteomics reveals myoferlin as a novel prognostic predictor in pancreatic adenocarcinoma. J Proteomics 91: 453-465, 2013. PMID: 23851313. DOI: $10.1016 /$ j.jprot.2013.06.032

11 Song DH, Ko GH, Lee JH, Lee JS, Yang JW, Kim MH, An HJ, Kang MH, Jeon KN and Kim DC: Prognostic role of myoferlin expression in patients with clear cell renal cell carcinoma. Oncotarget 8: 89033-89039, 2017. PMID: 29179496. DOI: 10.18632/oncotarget.21645

12 Jung M, Lee C, Park JH and Moon KC: Prognostic significance of immunohistochemical staining for myoferlin in clear cell renal cell carcinoma and its association with epidermal growth factor receptor expression. Urol Oncol 37: 812.e9-812.e16, 2019. PMID: 31421995. DOI: 10.1016/j.urolonc.2019.07.002

13 Zhang W, Zhou P, Meng A, Zhang R and Zhou Y: Downregulating myoferlin inhibits the vasculogenic mimicry of melanoma via decreasing MMP-2 and inducing mesenchymalto-epithelial transition. J Cell Mol Med 22: 1743-1754, 2018. PMID: 29164766. DOI: 10.1111/jcmm.13455

14 Rademaker G, Costanza B, Bellier J, Herfs M, Peiffer R, Agirman F, Maloujahmoum N, Habraken Y, Delvenne P, Bellahcene A, Castronovo V and Peulen O: Human colon cancer cells highly express myoferlin to maintain a fit mitochondrial network and escape p53-driven apoptosis. Oncogenesis 8: 21, 2019. PMID: 30850580. DOI: 10.1038/ s41389-019-0130-6

15 Bernatchez PN, Acevedo L, Fernandez-Hernando C, Murata T, Chalouni C, Kim J, Erdjument-Bromage H, Shah V, Gratton JP, McNally EM, Tempst P and Sessa WC: Myoferlin regulates vascular endothelial growth factor receptor-2 stability and function. J Biol Chem 282: 30745-30753, 2007. PMID: 17702744. DOI: $10.1074 /$ jbc.M704798200

16 Dupasquier S, Delmarcelle A-S, Marbaix E, Cosyns J-P, Courtoy PJ and Pierreux CE: Validation of housekeeping gene and impact on normalized gene expression in clear cell renal cell carcinoma: Critical reassessment of $\mathrm{YBX} 3 / \mathrm{ZONAB} / \mathrm{CSDA}$ expression. BMC Mol Biol 15: 9, 2014. PMID: 24885929. DOI: 10.1186/1471-2199-15-9

17 Jung M, Ramankulov A, Roigas J, Johannsen M, Ringsdorf M, Kristiansen $\mathrm{G}$ and Jung $\mathrm{K}$ : In search of suitable reference genes for gene expression studies of human renal cell carcinoma by real-time PCR. BMC Mol Biol 8: 47, 2007. PMID: 17559644. DOI: $10.1186 / 1471-2199-8-47$

18 Redpath GMI, Sophocleous RA, Turnbull L, Whitchurch CB and Cooper ST: Ferlins show tissue-specific expression and segregate as plasma membrane/late endosomal or trans-golgi/recycling ferlins. Traffic 17: 245-266, 2016. PMID: 26707827. DOI: $10.1111 /$ tra. 12370

19 UniProt: a worldwide hub of protein knowledge. Nucleic Acids Res 47: D506-D515, 2019. PMID: 30395287. DOI: 10.1093/ nar/gky 1049

20 An HJ, Song DH, Koh HM, Kim Y-M, Ko GH, Lee J-H, Lee JS, Yang JW, Kim MH, Seo DH, Jang SM and Kim DC: Myoferlin silencing inhibits VEGFR2-mediated proliferation of metastatic 
clear cell renal cell carcinoma. Sci Rep 9: 12656, 2019. PMID: 31477752. DOI: 10.1038/s41598-019-48968-7

21 Song DH, Ko GH, Lee JH, Lee JS, Lee G-W, Kim HC, Yang JW, Heo RW, Roh GS, Han S-Y and Kim DC: Myoferlin expression in non-small cell lung cancer: Prognostic role and correlation with VEGFR-2 expression. Oncol Lett 11: 998-1006, 2016. PMID: 26893682. DOI: 10.3892/ol.2015.3988

22 Fahmy K, Gonzalez A, Arafa M, Peixoto P, Bellahcene A, Turtoi A, Delvenne P, Thiry M, Castronovo V and Peulen O: Myoferlin plays a key role in VEGFA secretion and impacts tumorassociated angiogenesis in human pancreas cancer. Int $\mathrm{J}$ cancer 138: 652-663, 2016. PMID: 26311411. DOI: 10.1002/ijc.29820

23 Yu C, Sharma A, Trane A, Utokaparch S, Leung C and Bernatchez $\mathrm{P}$ : Myoferlin gene silencing decreases TIE-2 expression in vitro and angiogenesis in vivo. Vascul Pharmacol 55: 26-33, 2011. PMID: 21586340. DOI: 10.1016/j.vph.2011.04.001

24 Maisonpierre PC, Suri C, Jones PF, Bartunkova S, Wiegand SJ, Radziejewski C, Compton D, McClain J, Aldrich TH, Papadopoulos N, Daly TJ, Davis S, Sato TN and Yancopoulos GD: Angiopoietin-2, a natural antagonist for TIE2 that disrupts in vivo angiogenesis. Science 277: 55-60, 1997. PMID: 9204896. DOI: $10.1126 /$ science. 277.5322 .55
25 Steffens S, Schrader AJ, Blasig H, Vetter G, Eggers H, Trankenschuh W, Kuczyk MA and Serth J: Caveolin 1 protein expression in renal cell carcinoma predicts survival. BMC Urol 11: 25, 2011. PMID: 22152020. DOI: 10.1186/1471-2490-11-25

26 Ruan H, Li X, Yang H, Song Z, Tong J, Cao Q, Wang K, Xiao W, Xiao H, Chen X, Xu G, Bao L, Xiong Z, Yuan C, Liu L, Qu Y, $\mathrm{Hu}$ W, Gao Y, Ru Z, Chen K and Zhang X: Enhanced expression of caveolin-1 possesses diagnostic and prognostic value and promotes cell migration, invasion and sunitinib resistance in the clear cell renal cell carcinoma. Exp Cell Res 358: 269-278, 2017. PMID: 28684115. DOI: 10.1016/j.yexcr.2017.07.004

27 Wang X, Bullock AJ, Zhang L, Wei L, Yu D, Mahagaokar K, Alsop DC, Mier JW, Atkins MB, Coxon A, Oliner J and Bhatt RS: The role of angiopoietins as potential therapeutic targets in renal cell carcinoma. Transl Oncol 7: 188-195, 2014. PMID: 24704536. DOI: 10.1016/j.tranon.2014.02.003

Received April 5, 2020

Revised April 15, 2020

Accepted April 21, 2020 